\title{
EFFECT OF KINESIOLOGY TAPING ON DYSKINETIC SCAPULA IN INDIVIDUALS WITH ROTATOR CUFF DISORDERS
}

\section{Divya Kawediya ${ }^{1}$, Ketki Thosar ${ }^{2}$, Prajakta Sahasrabudhe ${ }^{3}$, Nilima Bedekar ${ }^{4}$.}

Postgraduate student, Sancheti Institute College of Physiotherapy, Pune, Maharashtra, India.

DPT Student, Touro College and University, New York City, United States.

Assistant Professor, Sancheti Institute College of Physiotherapy, Pune, Maharashtra, India.

Professor and Head of Musculoskeletal Department, Sancheti Institute College of Physiotherapy, Pune, Maharashtra, India.

\section{ABSTRACT}

Background: Rotator cuff being an important shoulder stabilizer and mobilizer, rotator cuff pathologies and subacromial impingement are among the most common shoulder disorders. Studies showed that there is early contraction of upper trapezius during abduction movement leading to trapezius muscle imbalance in rotator cuff disorders, resulting in altered scapula-humeral rhythm. Kinesiology taping (KT) techniques have several known benefits. Kase et al have proposed several benefits, depending on the amount of stretch applied to the tape during application viz. to provide a positional stimulus through the skin, to align fascia, to create more space by lifting fascia and soft tissue above area of pain or inflammation, to provide sensory stimulation to assist or limit motion, to assist in the removal of oedema.

Materials and Methods: Three groups were formed viz. group A, group B (facilitatory taping for lower trapezius and inhibitory taping for upper trapezius) and group $C$ (inhibitory taping for upper trapezius). 45 individuals fulfilling the inclusion and exclusion criteria were selected and randomly allocated in three groups. In this single blinded study, the second author measured the lateral scapular slide test on day 1 and day 4 . The subjects were asked to perform similar and limited exercises.

Results: Data analysis was done using paired t-test for comparing the pre and post values of scapular dyskinesia within the group and one way ANOVA was used for comparing the difference between the groups. The results of this study suggested that, although improvement is seen in the groupC, it is significantly enhanced with the muscle inhibitory and facilitatory effect of kinesiology tape seen in group $B(p$ value<.01).

Conclusion: Owing to the results and statistical analysis, the study concluded that both inhibitory and facilitatory technique of kinesiology tape application have a significant effect in reducing scapular dyskinesia in patients with rotator cuff disorders. Thus KT can be used as an adjunct to the exercise program while treating scapular dyskinesia in patients with rotator cuff disorders.

KEY WORDS: Kinesiology Taping, Scapular Dyskinesia, Rotator Cuff Disorders, Trapezius.

Address for correspondence: Dr. Divya Kawediya, Postgraduate student, Sancheti Institute College of Physiotherapy, Pune, Maharashtra, India. E-Mail: diya.0512@gmail.com

Access this Article online

Quick Response code

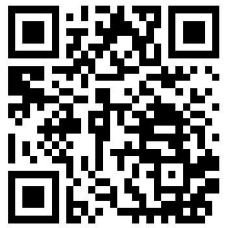

DOI: $10.16965 /$ ijpr.2018.146

Journal Information

International Journal of Physiotherapy and Research

ICV for 2016 ISSN (E) 2321-1822 | ISSN (P) 2321-8975

86.93

https://www.ijmhr.org/ijpr.html

DOI-Prefix: https://dx.doi.org/10.16965/ijpr

Article Information

Received: 28 Apr 2018

Peer Review: 28 Apr 2018

Revised: None
Accepted: 25 May 2018

Published (O): 11 June 2018

Published (P): 11 June 2018

\section{INTRODUCTION}

The stability of the shoulder girdle is provided by an intricate balance between the scapular and gleno-humeral muscles and the structures of the joints in the girdle. The rotator cuff muscles play an integral role in the shoulder movement. The rotator cuff group As the osteokinematic and arthrokinematic motions of 
the humeral head in the glenoid and along with the biceps depresses the humeral head during movements into elevation [1]. Rotator cuff disorders include:

- Inflammation of the tendons (tendinitis) or of a bursa (bursitis)

- Impingement,

- Calcium buildup in the tendons, which causes a painful condition called calcific tendinitis.

- Partial or complete tear of the rotator cuff [2] Rotator cuff pathology and sub-acromial impingement are among the most common diagnosis made in the shoulder region. EMG studies have shown that there occurs trapezius muscle imbalance in individuals suffering from rotator cuff disorders. Early contraction of upper trapezius is noted especially during flexion and abduction movements in these individuals, resulting in altered scapula-humeral rhythm [3]. Scapular dyskinesia is one of the common symptoms. Biomechanical research outlining the complex interaction between the scapulothoracic and glenohumeral joints during humeral elevation has resulted in descriptive data and identified consistent patterns of motion and interrelated movements between the scapula and humerus[4,5] Upward rotation, external rotation, and posterior tilting of the scapula are reportedly necessary for successful humeral elevation $[4,5,6]$. These movements, although objectively measurable in the laboratory setting with complex instrumentation such as three-dimensional tracking $[4,5,6]$, are typically evaluated by clinicians in patients with shoulder dysfunction using only visual observation. Various authors $[7,8]$ defined scapular dyskinesis as "an observable alteration of the position and motion of the scapula relative to the thoracic cage." Vast majority of these cases are initially treated non-operatively. This places physical therapy as a likely first line of treatment for this problem.

In recent years, the use of kinesiology tape (KT) has become increasingly popular. KT is roughly the same thickness as the epidermis and can be stretched $30 \%-40 \%$ of its resting length longitudinally. Kase et al have proposed several benefits, depending on the amount of stretch applied to the tape during application viz. to provide a positional stimulus through the skin, to align fascia, to create more space by lifting fascia and soft tissue above area of pain or inflammation, to provide sensory stimulation to assist or limit motion, to assist in the removal of oedema[9]. KT is latex free and the adhesive is $100 \%$ acrylic and heat activated. Prescribed wear time for 1 application is longer, usually 3-4 days[9].

The normal scapula-humeral rhythm is crucial for performing shoulder movements. In majority of the shoulder pathologies, there is altered scapula-humeral rhythm due to early contraction of upper trapezius during flexion and abduction of the shoulder. This leads us to the need for the study so as to study the effect of inhibitory kinesiology taping on upper trapezius and facilitatory kinesiology taping on lower trapezius during shoulder abduction movement in individuals with rotator cuff disorders with dyskinetic scapula. Thus the aim of this research was to study the effect of kinesiology taping on dyskinetic scapula in patients with rotator cuff disorders. The objectives of this research were to study -

a) the effect of inhibitory KT for upper trapezius and facilitatory taping for lower trapezius (Group B)

b) the effect of inhibitory KT for upper trapezius (Group C)

on early scapular elevation during shoulder abduction movement measured by Lateral Scapular Slide Test[10]. And to compare the readings of lateral scapular slide test in between group $B$, group $C$ and group $A$ group.

\section{MATERIALS AND METHODS}

In this single blinded randomized control trial 45 individuals with rotator cuff disorders with dyskinetic scapula between $25-50$ years of age were selected. Individuals with frozen shoulder, complete tear of the rotator cuff, acute pain in the shoulder region and those with any trauma around the shoulder girdle were excluded from this study. Informed consent was obtained from all subjects before enrollment and all rights of the subjects were protected. Using random allocation subjects were divided into 3 groups. To avoid bias the second author, who was blinded to the group assignment, measured the lateral 
scapular slide test on day 1 and again on day 4.

Lateral scapular slide test [10] -The test was performed in the standing position. The subjects were adequately exposed. With the scapula in the normal resting position and the arms by the side of the body(0 degrees abduction) markers were applied on the following bony landmarks on both the right and left side:

-root of the spine of the scapula and the corresponding spinous process

-inferior angle of the scapula and the corresponding spinous process

-mid-point of the root and inferior angle of the scapula and the corresponding spinous process.

The distance between the root of the spine of the scapula and the corresponding spinous process(D1), mid-point of the root and inferior angle of the scapula and the corresponding spinous process(D2), inferior angle of the scapula and the corresponding spinous process(D3) were measured in $\mathrm{mm}$ and recorded on both the sides using a digital vernier caliper.

For upper trapezius taping the patient was adequately exposed. The area to be taped was sterilized. The tape was cut to an appropriate length and the edges were rounded. Upper trapezius was stretched by side flexing the neck to the opposite side of the muscle to be taped. The anchor was placed on the acromion process. The muscle was taped from Insertion-Origin using paper-off tension (10-15\% stretch) for inhibition along the muscle length. The tape was rubbed to activate the glue and then the stretch on the muscle was relieved[9].

Whereas for lower trapezius taping the above procedure was repeated. Lower trapezius was stretched by doing horizontal adduction. The anchor of the tape was placednear the origin. Further, using a Y-shaped tape, giving 25-35\% stretch, both tails were placed in such a way as to cover the entire muscle tissue from origin to insertion. The tape was rubbed adequately and glue was activated. Finally the stretch on the muscle was relieved[9].

In the Group A group the subjects were given strengthening protocol for Rhomboids, Middle and Lower trapezius, Rotator cuff viz. Supraspinatus, Infraspinatus, Subscapularis and Teres minor, Serratus anterior. 10 repetitions*1 set using red theraband.

For the subjects in group B facilitatory taping for lower trapezius and inhibitory taping for lower trapezius was given in addition to the above mentioned strengthening protocol. The subjects in group $\mathrm{C}$ were given only inhibitory taping for upper trapezius along with the strengthening protocol.

The Lateral Scapular Slide Test was measured on day 1 and on day 4 immediately after the removal of the tape. The data was collected and further analyzed using paired t-test for within group analysis and single-factor ANOVA for between group analysis.

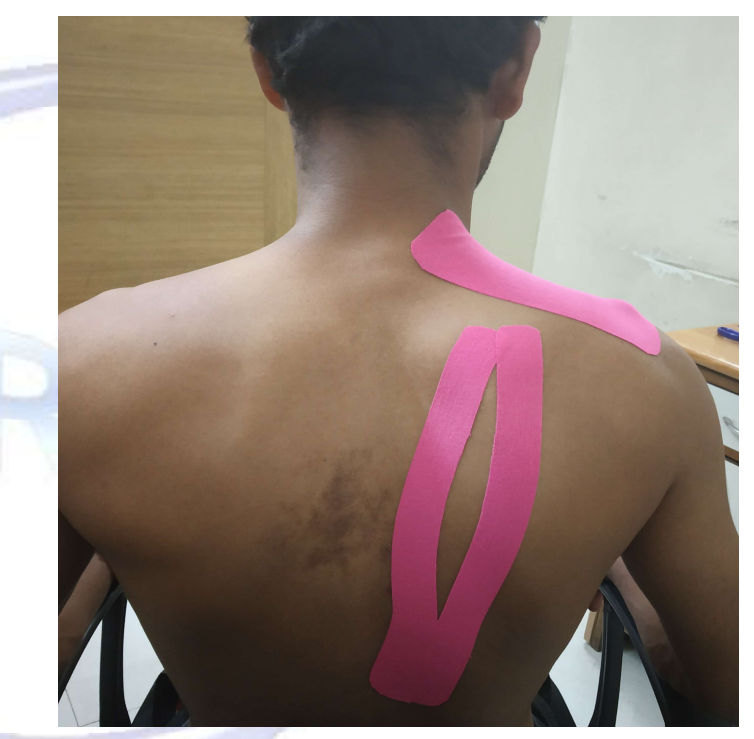

\section{RESULTS}

Data analysis was done using SPSS version 16, paired t-test for comparing the pre and post values of scapular dyskinesia within the group and One way ANOVA was used for comparing the difference between the three groups.

Table 1: LSST values.

\begin{tabular}{|c|c|c|c|c|}
\hline & & \multicolumn{3}{|c|}{ P Value } \\
\hline Angle & Level & Group A & Group B & Group C \\
\hline \multirow{4}{*}{0} & Spine & $>.05$ & $<.05^{*}$ & $<.05^{*}$ \\
\cline { 2 - 5 } & Mid & $>.05$ & $<.05^{*}$ & $<.05^{*}$ \\
\cline { 2 - 5 } & Inferior & $>.05$ & $<.05^{*}$ & $<.05^{*}$ \\
\hline \multirow{4}{*}{$\mathbf{4 5}$} & Spine & $>.05$ & $<.05^{*}$ & $<.05^{*}$ \\
\cline { 2 - 5 } & Mid & $>.05$ & $<.05^{*}$ & $<.05^{*}$ \\
\cline { 2 - 5 } & Inferior & $>.05$ & $<.05^{*}$ & $<.05^{*}$ \\
\hline \multirow{4}{*}{$\mathbf{9 0}$} & Spine & $>.05$ & $<.05^{*}$ & $>.05$ \\
\cline { 2 - 5 } & Mid & $>.05$ & $<.05^{*}$ & $<.05^{*}$ \\
\cline { 2 - 5 } & Inferior & $>.05$ & $>.05$ & $>.05$ \\
\hline \multirow{3}{*}{$\mathbf{1 2 0}$} & Spine & $>.05$ & $<.05^{*}$ & $<.05^{*}$ \\
\cline { 2 - 5 } & Mid & $>.05$ & $<.05^{*}$ & $<.05^{*}$ \\
\cline { 2 - 5 } & Inferior & $>.05$ & $<.05^{*}$ & $>.05$ \\
\hline
\end{tabular}


Intra-Group Analysis: As seen in Table 1

For group A- The pre-post differences at 0, 45, 90, 120 degrees of scapular abduction when measured at spine, mid-point and inferior angle of scapula were not significant ( $p$ value $>.05$ ).

For group B- The pre-post differences at 0 and 45 degrees of scapular abduction when measured at all three levels showed a significant improvement ( $p$ value< .05 ). Although the differences at 90 degrees were significant at spine and mid scapula, the improvement was not significant enough at the inferior angle. At 120 degrees of scapular abduction, the improvement was statistically significant ( $p$ values $<.05$ )

For group C- The pre-post differences at 0 and 45 degrees of scapular abduction when measured at all three levels showed a significant improvement ( $p$ value $<.05$ ) Although the differences at 90 degrees were significant at mid scapula, the improvement was not significant enough at spine and inferior angle. At 120 degrees of scapular abduction, the improvement was statistically significant ( $p$ values $<.05$ ) at spine and mid scapula with an exception of inferior angle ( $p$ value $>.05$ )

\section{Intergroup Analysis:}

At $0,45,90,120$ degrees of scapular abduction the comparison between the group $A$, group $B$ and group $C$ were highly significant ( $p$ value $<.01$ ) when measured at spine, mid-point and inferior angle of scapula as seen in Table 1. Group B was found to be better compared to Group $C$ and Group A as seen in Figure 1,2, 3 and 4.

Fig. 1: Graph showing pre-post difference of LSST at Op of abduction.



Int J Physiother Res 2018;6(3):2777-82. I ISSN 2321-1822
Fig. 2: Graph showing pre-post difference of LSST at $45 p$ of abduction.

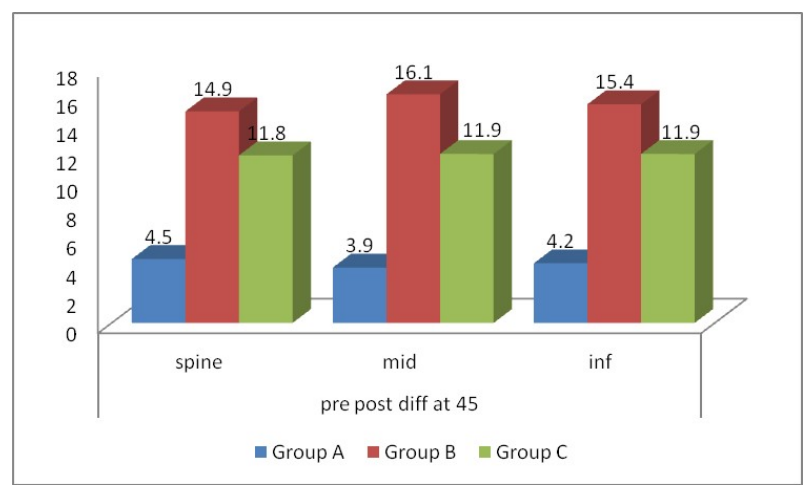

Fig. 3: Graph showing pre-post difference of LSST at 90p of abduction.

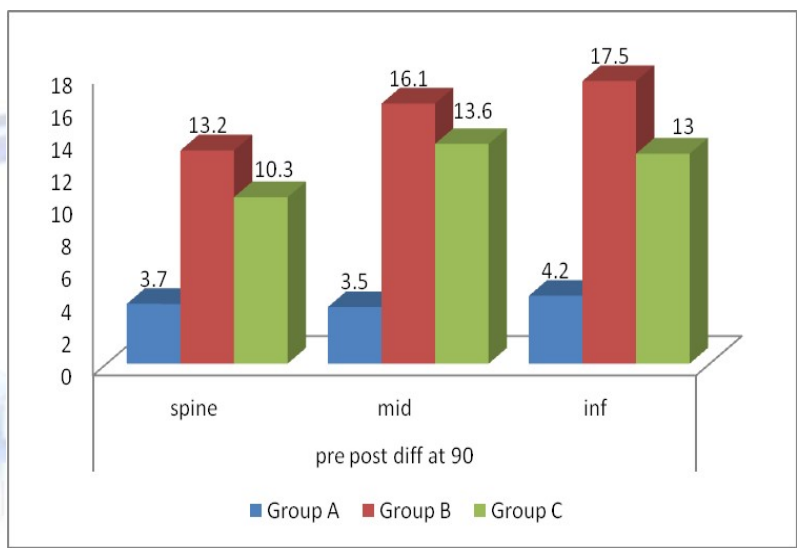

Fig. 4: Graph showing pre-post difference of LSST at 120p of abduction.

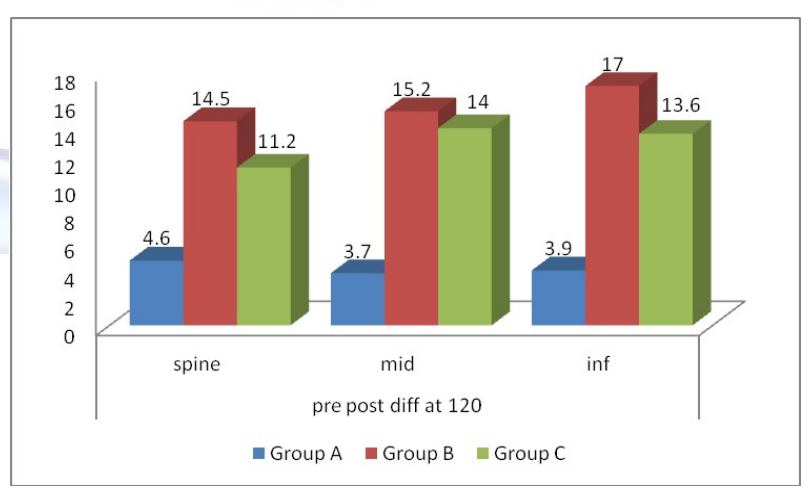

\section{DISCUSSION}

Scapular dyskinesia is an alteration in the normal position or motion of scapula during coupled scapula-humeral movements. With faulty scapular posture, muscle length and strength imbalances occur not only in the scapular muscles but also in the humeral muscles, thus, altering the mechanics of gleno-humeral joint $[11,12]$.

The subjects included in this study were from type III: superior border prominence indicating over activity of levator scapulae and upper trapezius along with imbalance of the upper and lower trapezius force couple associated with 
impingement and rotator cuff lesions [4].

A study discussed the mechanical, proprioceptive and neuromuscular effects of taping being able to influence the length of muscle fibers, inducing a shift of the length-tension curve of those muscles and consequently increasing the overlap between the actin and myosin filaments and therefore cross-bridge formation. A significant increase in the afferent stimuli reaching the CNS was recorded through taping-induced cutaneous stimulation thus increasing the proprioceptive stimulus. The neuromuscular mechanism has its substrate of action within the spinal cord, where inter neuronal connections which are able to alter the state of muscle contraction are stimulated by an increased sensory stimulus coming from the skin. The results of this study show a significant effect of the taping application with facilitatory meaning, while the inhibitory intervention lacked significance [13]. This supports the hypothesis of this study that kinesiology taping is effective in treating patients. Although inhibitory taping lacked significant results in the mentioned study, it could have been because of 'neutralization' which states that the muscular effects of this would be observable only in the presence of a pre-existing change in tone due to a biomechanical dysfunction or pain.

In this study, the effect of inhibitory and facilitatorykinesio taping on improvement in scapular dyskinesia was noted and measured using the lateral scapular slide test. The result indicated maximum improvement in scapular dyskinesia in group $B$ subjects. This signified that usage of taping method augmented the exercise program given to the patient. Studies have reported that there is over activity of upper trapezius in patients with rotator cuff disorders, as a compensatory strategy to facilitate abduction at the gleno humeral joint. The inhibitory KT application used for upper trapezius possibly helped in reducing the over firing of motor units of the muscle. The facilitatory technique used for lower trapezius might have helped in recruiting more motor units to perform the muscle action due to an increased proprioceptive stimulus [13]. The continuous proprioceptive feedback helped in better positioning of scapula and improving the arc of motion in which the scapula abducts.

There was a study conducted to investigate the association between sub-acromial symptoms and upper and lower trapezius imbalance and to find out the effectiveness of scapular taping on muscle activity, the results showed a significant difference in reducing the upper trapezius activity but not much with lower trapezius taping [14]. But in this study, both upper and lower trapezius muscle fibers were taped and a cumulative effect was observed. The lateral scapular slide test was performed before and after the tape application and not while the tape was in situ. A significant difference was seen in groups B and C. Group B showed a significant improvement probably because the lowered upper trapezius activity helped in stabilizing the scapula. This, in turn, helped in better strengthening of scapular stabilizers.

Various researches done to study effect of KT concluded that Selkowitz DM et al showed that $\mathrm{KT}$ application in healthy individuals improves muscle activation through positive mechanical, proprioceptive and neuromuscular effects improving length tension relationship of muscle fibers and hence facilitatory effect [3].

KT application for facilitation of lower trapezius in subacromial impingement had shown improved recruitment pattern of the muscle fibers on EMG [4]. The result of another study showed a significant difference in reducing the upper trapezius activity. It showed that upper trapezius motor unit recruitment was deceased with inhibitory taping [3].

Overhead shoulder abduction requires coordinated action of scapular muscles. When overactive trapezius was inhibited using KT, scapular dyskinesia was found to be reduced on LSST possibly due to reduced firing of motor units supplying upper trapezius. When facilitatory taping was added further improvement was noticed in scapular dyskinesia. This can be attributed to improved recruitment of lower trapezius fibers and hence more coordinated movement of scapula.

\section{CONCLUSION}

Owing to the statistical analysis and results obtained, it can be concluded that both inhibitory and facilitatory technique of kinesiology 
tape application has a significant effect in reducing scapular dyskinesia in patients with rotator cuff disorders. Thus KT can be used as an adjunct to the exercise program while treating scapular dyskinesia in patients with rotator cuff disorders.

\section{ABBREVIATIONS}

\section{KT- Kinesiology Taping}

LSST- Lateral Scapular Slide Test

\section{ACKNOWLEDGEMENTS}

We are grateful to all the participants for providing for their valuable time for this study. A heartfelt thank you to Dr. RachanaDabadghav (PT) for conducting the statistical analysis andDr.AnkitSrivastava (PT) for his constant support and motivation.

\section{Conflicts of interest: None}

\section{REFERENCES}

[1]. Levangie PK, Norkin CC. Joint structure and function: a comprehensive analysis. FA Davis; 2011 Mar 9.

[2]. Huijbregts PA, Bron C. Rotator cuff lesions: shoulder impingement. Manual Therapy for Musculoskeletal Pain Syndromes E-Book: an evidence-and clinicalinformed approach. 2015 Apr;28:309.

[3]. Selkowitz DM, Chaney C, Stuckey SJ, Vlad G. The effects of scapular taping on the surface electromyographic signal amplitude of shoulder girdle muscles during upper extremity elevation in individuals with suspected shoulder impingement syndrome. journal of orthopaedic \& sports physical therapy. 2007 Nov;37(11):694-702.

[4]. Ellenbecker TS, Kibler WB, Bailie DS, Caplinger R, Davies GJ, Riemann BL. Reliability of scapular classification in examination of professional baseball players. Clinical Orthopaedics and Related Research $^{\circledR}$. 2012 Jun 1;470(6):1540-4.
[5]. Winter DA. Biomechanics and motor control of human movement. John Wiley \& Sons; 2009 Oct 12.

[6]. Schmidt RA, Lee T, Winstein C, Wulf G, Zelaznik H. Motor Control and Learning, 6E. Human kinetics; 2018.

[7]. Manske R, Ellenbecker T. Current concepts in shoulder examination of the overhead athlete. International journal of sports physical therapy. 2013 Oct;8(5):554.

[8]. Kibler BW, McMullen J. Scapular dyskinesis and its relation to shoulder pain. JAAOS-Journal of the American Academy of Orthopaedic Surgeons. 2003 Mar 1;11(2):142-51.

[9]. KenzoKase, Jim Wallis, Tsuyoshi Kase; Clinical Therapeutic Applications Of The Kinesio Taping Method; $2^{\text {nd }}$ Edition

[10]. Magee DJ. Orthopedic physical assessment-E-Book. Elsevier Health Sciences; 2014 Mar 25.

[11]. Kisner C, Colby LA, Borstad J. Therapeutic exercise: foundations and techniques. Fa Davis; 2017 Oct 18.

[12]. Kibler WB, Sciascia A. Current concepts: scapular dyskinesis. British journal of sports medicine. 2010 Apr 1;44(5):300-5.

[13]. Gusella A, Bettuolo M, Contiero F, Volpe G. Kinesiologic taping and muscular activity: a myofascial hypothesis and a randomised, blinded trial on healthy individuals. Journal of bodywork and movement therapies. 2014 Jul 1;18(3):405-11.

[14]. Smith M, Sparkes V, Busse M, Enright S. Upper and lower trapezius muscle activity in subjects with subacromial impingement symptoms: is there imbalance and can taping change it?. Physical Therapy in Sport. 2009 May 1;10(2):45-50.

\footnotetext{
How to cite this article:

Divya Kawediya, Ketki Thosar, Prajakta Sahasrabudhe, Nilima Bedekar. EFFECT OF KINESIOLOGY TAPING ON DYSKINETIC SCAPULA IN INDIVIDUALS WITH ROTATOR CUFF DISORDERS. Int J Physiother Res 2018;6(3):27772782. DOI: $10.16965 /$ ijpr.2018.146
} 